\title{
Unilaterales polyradikuläres Defizit von C4 bis C6 als Symp- tom einer spontanen extrakraniellen Vertebralisdissektion
}

\section{Warum dieser Fall?}

Der Fall beschreibt als seltene Ursache einer schmerzhaften peripheren hochgradigen proximalen sensomotorischen Armparese eine extrakranielle Vertebralisdissektion. Der neurologische Untersuchungsbefund und das initial native HWS-MRT sowie in der Folge die spezielle MR-Untersuchung zur Frage Vertebralisdissektion führten zur Diagnose.

\section{Einleitung \\ $\nabla$}

Unilaterale schmerzhafte periphere proximale sensomotorische Armparesen können z.B. durch eine obere Armplexusläsion bedingt sein. Ursachen sind hier u.a. Trauma, Entzündung, neoplastische Raumforderung oder radiogene Plexusschädigung. Ebenso können eine Polyradikulitis oder kompressive Radikulopathien vorliegen, verursacht z.B. durch degenerative Veränderungen der HWS mit Einengung der Foramina intervertebralia oder Bandscheibenvorfälle. Eine ipsilaterale Vertebralisdissektion als Ursache hingegen ist äußerst selten.

\section{Fallbericht}

\section{$\nabla$}

Ein 47-jähriger Patient stellte sich in der Zentralen Notaufnahme vor mit seit ca. 10 Tagen bestehenden Schmerzen im rechten Nacken- und Schulterbereich bis in den Hinterkopf rechts ausstrahlend. Seit 5 Tagen bestand bei unverändertem Schmerz eine Schwäche der Schulterund Oberarmmuskulatur rechts, welche im Verlauf progredient war. Daneben berichtete der Patient über ein Taubheitsgefühl im Schulter- und im oberen Brustbereich rechts.

Im Neurostatus zeigte sich eine manifeste proximale Armparese rechts (M. deltoideus KG3/5, M. biceps brachii KG4/5, M. supraspinatus KG 4/5, M. infraspinatus KG 3/5). Der Bizepssehnenreflex war rechts abgeschwächt. Hypästhesie und Hypalgesie unterhalb der Klavikula rechts und an der Schulteraußenseite rechts dem C4- und C5-Dermatom entspre- chend. Darüber hinaus unauffälliger neurologischer Untersuchungsbefund, insbesondere kein Horner.

Im notfallmäßig durchgeführten MRT der HWS nativ ergab sich kein Anhalt für eine polysegmentale Einengung der Foramina intervertebralia durch Bandscheibenvorfall oder degenerativ knöcherne Veränderungen. Dagegen wurde der dringende Verdacht auf eine Dissektion der Arteria vertebralis rechts geäußert ( $\bullet$ Abb. 1).

Der Patient wurde daraufhin gewichtsadaptiert mit Clexane antikoaguliert und dann auf Marcumar mit einem Ziel-INR von 2,0-3,0 eingestellt, empfohlen für ca. 6 Monate. In der weiteren Diagnostik ergab die speziell auf die Frage einer Vertebralisdissektion ausgerichtete MR-Diagnostik im MRT des Halses ( $\bullet$ Abb.2a u. b) den Nachweis einer Vertebralisdissektion

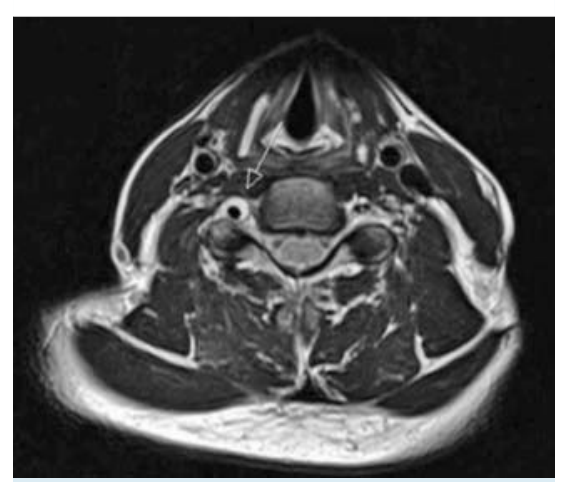

Abb.1 Im nativ HWS-MRT axial V.a. Dissektion $\operatorname{der} A$. vertebralis rechts.

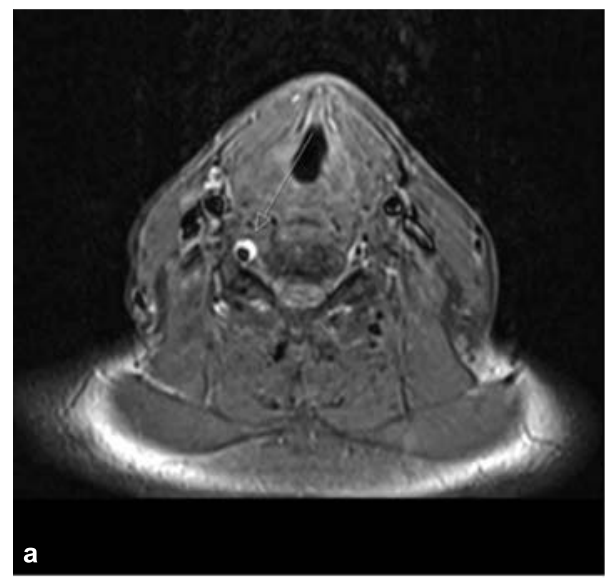

Abb. 2a, b Nachweis einer langstreckigen Dissektion der extrakraniellen A. vertebralis rechts im MRTBild mit besserer Darstellung des Dissekats durch Einsatz der fettsupprimierten T1-Wichtung.

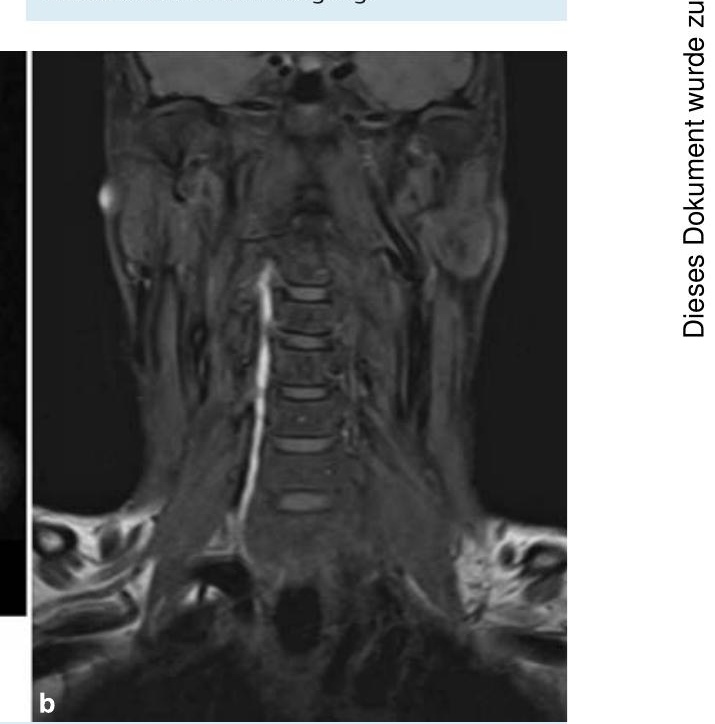

rechts mit langstreckigem Wandhämatom der extrakraniellen A. vertebralis rechts bis zur Atlasschlinge reichend ohne Lumeneinengung in der MR-Angio des Halses ( $\bullet$ Abb.3).

Das zusätzlich durchgeführte cMRT bot keinen Anhalt für eine zerebrale Ischämie. Die weitere Diagnostik lieferte keine Hinweise für Gefäßerkrankungen, sodass von einer spontanen extrakraniellen Vertebralisdissektion rechts auszugehen ist.

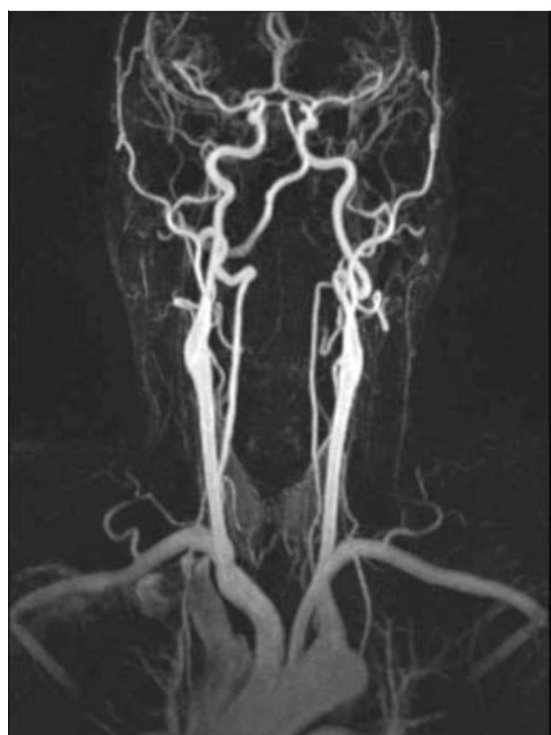

Abb. 3 In der MR-Angio Hals A. vertebralis rechts ohne Lumeneinengung. 


\section{Diskussion}

\section{$\nabla$}

Dissektionen, spontan oder traumatisch bedingt, sind häufig Ursache von zerebralen Ischämien. Typisch für eine Vertebralisdissektion ist z.B. das Wallenberg-Syndrom bedingt durch einen direkten Verschluss der A. cerebelli inferior posterior (PICA) [1]. Andere klinische Zeichen der Vertebralisdissektion sind einseitige Nackenschmerzen mit Ausstrahlung in den Arm und nach okzipital. Bei intraduraler Lage können sie eine SAB verursachen. Als Ursache von C4-, C5- und C6-Wurzelläsionen sind Vertebralisdissektionen eine Rarität [2, 3].

Dissektionen treten meist einseitig an Prädilektionsstellen auf. Dies sind Stellen mit erhöhter mechanischer Beanspruchung. Bei der A. vertebralis ist dies das V1-Segment, unmittelbar am Eintritt der A. vertebralis in den Querfortsatz meist des 6. Halswirbels, und das V3-Segment, die Atlasschlinge.

Man nimmt an, dass extrakranielle Dissektionen primär durch eine Einblutung aus den Vasa vasorum in die äußeren Schichten der Arterienwand entstehen, also Einblutungen in die Tunica media oder zwischen Tunica media und Tunica adventitia [4-6], sog. äußere Dissektion. Dies führt zu einer Erweiterung des Gefäßes nach außen mit lokaler Kompression der in enger topografischer Nachbarschaft liegenden Nervenwurzeln [7]. Außerdem kann es hierdurch zu Durchblutungsstörungen benachbarter Strukturen wie z. B. zervikaler Nervenwurzeln kommen.

Davon unterschieden werden innere Dissektionen mit Einblutungen zwischen Tunica intima und Tunica media mit möglicher Lumeneinengung bis hin zum Gefäßverschluss. Dabei steigt das Risiko von Hirninfarkten mit dem Stenosierungsgrad [8]. Es kommt hierbei zu von der geschädigten Intima ausgehenden Embolien oder zu Beeinträchtigungen der Hämodynamik.

Die Diagnose einer Dissektion wird über den Nachweis des intramuralen Hämatoms im MRT in den hochauflösenden T1-gewichteten Sequenzen mit Fettsuppression gestellt [9-13]. Der pathognomische Befund ist ab dem 2. -4 . Tag nach Dissektionsbeginn nachzuweisen und persistiert über mehrere Wochen.

Die Empfehlungen zur Behandlung einer Dissektion sind uneinheitlich. Unsere Behandlung bestand in der Einleitung einer oralen Antikoagulation mit Marcumar für ca. 6 Monate (Ziel-INR 2,0-3,0). Laut Leitlinie ist diese Therapie nicht evidenzbasiert. Prospektive randomisierte Studien zur Über- oder Unterlegenheit gegenüber der Therapie mit Thrombozytenaggregationshemmern liegen nicht vor [14]. Die Behandlung der extrakraniellen Vertebralisdissektion kann deshalb mit oraler Antikoagulation [15] oder mit Thrombozytenaggregationshemmung [16] erfolgen. Bezüglich einer C5/C6-Radikulopathie bei Vertebralisdissektion ist im Einzelfall auch eine operative Dekompression der entsprechenden Nervenwurzeln beschrieben [17].

\section{Zusammenfassung}

Ein Patient mit persistierenden SchulterNacken-Schmerzen rechts und wenige Tage später auftretender peripherer hochgradiger proximaler sensomotorischer Armparese mit Defiziten von C4-C6 rechts ist zunächst verdächtig auf eine obere Armplexusläsion, nachrangig auf Radikulopathien z.B. verursacht durch degenerative HWS-Veränderungen. Im vorliegenden Fall wurde in der MR-Bildgebung als seltene Ursache eine extrakranielle ipsilaterale Vertebralisdissektion nachgewiesen.

\section{Was lernt man daraus?}

Eine schmerzhafte periphere proximale sensomotorische Armparese erfordert eine sorgfältige differenzialdiagnostische Abklärung. Sollten sich weder Hinweise für eine kompressive oder entzündliche Armplexusläsion noch für Radikulopathien bei degenerativen HWS-Veränderungen oder entzündlich bedingte Wurzelläsionen ergeben, sollte an die seltene Ursache einer extrakraniellen Vertebralisdissektion als Ursache gedacht werden, und eine entsprechende MR-Bildgebung zu dieser Frage erfolgen. Primär äußere Dissektionen ohne wesentliche Stenosierung des Gefäßes sind sonografisch schwer zu erkennen. Behandelt werden kann mit oraler Antikoagulation oder mit Thrombozytenaggregationshemmung.

Interessenkonflikt: Die Autoren geben an, dass kein Interessenkonflikt besteht.

\section{Literatur}

1 Sturzenegger M. Vertebralisdissektion. Klinik, nichtinvasive Diagnostik, Therapie - Beobachtungen an 14 Patienten. Nervenarzt 1994; 65: 402-410

2 Hetzel A, Berger W, Schumacher $M$ et al. Dissection of the vertebral artery with cervical nerve root lesions. J Neurol 1996; 243: 121 125

3 McGillion SF, Weston-Simons S, Harvey JR. Vertebral artery dissection presenting with multilevel combined sensorimotor radiculopathy: a case report and literature review. J Spinal Disord Tech 2009; 22: 456-458

4 Sasaki O, Ogawa H, Koike T et al. Clinicopathological study of dissecting aneurysms of the intracranial vertebral artery. J Neurosurg 1991; 75: 874-882

5 Müller BT, Luther B, Hort $W$ et al. Surgical treatment of 50 carotid dissections: indications and results. J Vasc Surg 2000; 31: 980-988

6 Völker W, Dittrich R, Grewe S et al. The outer arterial wall layers are primarily affected in spontaneous cervical artery dissection. Neurology 2011; 76: 1463-1471

7 Eberhardt O, Topka H. Compressive cervical radiculopathy due to vertebral artery dissection. J Stroke Cerebrovasc Dis 2015; 24: e115-116

8 Baumgartner RW, Arnold M, Baumgartner I et al. Carotid dissection with and without ischemic events: local symptoms and cerebral artery findings. Neurology 2001; 57: 827-832

9 Müllges W, Ringelstein EB, Leibold M. Non-invasive diagnosis of internal carotid artery dissections. J Neurol Neurosurg Psychiatry 1992; 55: 98 - 104

10 Kirsch E, Kaim A, Engelter $S$ et al. MR angiography in internal carotid artery dissection: improvement of diagnosis by selective demonstration of the intramural haematoma. Neuroradiology 1998; 40: 704-709

11 Fiebach J, Brand T, Knauth $M$ et al. MRI with fat suppression in the visualization of wall hematoma in spontaneous dissection of the internal carotid artery. Fortschr Röntgenstr 1999; 171: 290-293

12 Bachmann R, Nassenstein I, Kooijman $\mathrm{H}$ et al. Spontaneous acute dissection of the internal carotid artery: highresolution magnetic resonance imaging at 3.0 tesla with a dedicated surface coil. Invest Radiol 2006; 41: $105-$ 111

13 Bachmann R, Nassenstein I, Kooijman $\mathrm{H}$ et al. High resolution magnetic resonance imaging at 3.0 tesla in the short-term follow-up of patients with proven cervical artery dissection. Invest Radiol 2007; 42: 460 - 466

14 Engelter ST, Brandt T, Debette $S$ et al. for the Cervical Artery Dissection in Ischemic Stroke Patients (CADISP) Study Group. Antiplatelets versus anticoagulation in cervical artery dissection. Stroke 2007; 38: 2605 2611

15 Tabatabai G, Schöber W, Ernemann $U$ et al. Vertebral artery dissection presenting with ipsilateral acute C5 and C6 sensorimotor radiculopathy: A case report. Cases J 2008; 1: 139 
16 Silbert BI, Khangure M, Silbert PL. Vertebral artery dissection as a cause of cervical radiculopathy. Asian Spine J 2013; 7: 335-338

17 Kunert P, Prokopienko M, Czernicki $T$ et al. Sensorimotor C5 and C6 radiculopathy caused by thrombosed vertebral artery dissection and successfully treated with limited oblique corpectomy - Case report. Neurol Neurochir Pol 2016; 50: 48-51

\section{Unilateral Polyradicular Deficit from C4 to C6 as a Symptom of a Spontaneous Extracranial Verte- bral Dissection}

\section{Autoren}

K. Klemann ${ }^{1}$, C. Thurlow ${ }^{2}$, A. El Mountassir ${ }^{2}$, K. Wessel ${ }^{1}$

\section{Institute}

${ }^{1}$ Klinik für Neurologie, Städtisches Klinikum

Braunschweig

${ }^{2}$ Klinik für Radiologie, Städtisches Klinikum

Braunschweig

\section{Korrespondenzadresse}

\section{Dr. Katja Klemann}

Klinik für Neurologie,

Klinikum Braunschweig gGmbH

Salzdahlumer Straße 90

38126 Braunschweig

k.klemann@klinikum-braunschweig.de

\section{Bibliografie}

DOI http://dx.doi.org/

10.1055/s-0042-116289

Klin Neurophysiol 2016; 47: 215-217

(C) Georg Thieme Verlag KG

Stuttgart · New York

ISSN 1434-0275
Möchten Sie von einem besonderen Fall aus Ihrer Klinik berichten?

Autorenhinweise finden Sie unter:

http://www.thieme.de/de/klinische-neurophysiologie/ autoren-1869.htm

Schicken Sie Ihre Beiträge an: k.wessel@klinikum-braunschweig.de. 